\title{
A Hilbert Space Proof of the Fundamental Theorem of Asset Pricing in Finite Discrete Time
}

\author{
W. SCHACHERMAYER \\ Institut für Mathematik der Universität Wien, \\ Strudlhofgasse 4, A-1090 Wien, Austria.
}

October 92

\begin{abstract}
R. Dalang, A. Morton and W. Willinger have proved a beautiful version of the Fundamental Theorem of Asset Pricing which pertains to the case of finite discrete time: In this case the absence of arbitrage opportunities already characterizes the existence of an equivalent martingale measure.

The purpose of this paper is to give an elementary proof of this important theorem which relies only on orthogonality arguments. In contrast, the original proof of Dalang, Morton and Willinger uses heavy functional analytic machinery, in particular measurable selection and measure-decomposition theorems. We feel that the theorem (as well as its proof) should be accessible to a wider public and we therefore made an effort to keep the arguments as selfcontained as possible. In a final chapter we review and prove the necessary tools for our presentation of the theorem.
\end{abstract}

\section{Introduction}

We consider an $\mathbb{R}^{d}$-valued stochastic process $\left(S_{t}\right)_{t=0}^{N}$ which is indexed by the finite discrete time set $\{0,1, \ldots, N\}$. In mathamatical finance the process $S$ usually models the (discounted) price process of $d$ stocks.

The "Fundamental Theorem of Asset Pricing" states that the existence of an equivalent martingale measure for the process $S$ is "essentially" equivalent to the absence of arbitrage opportunities. The theorem is rightly termed "fundamental" as it allows to relate the concept of pricing by arbitrage - which has experienced increasing importance since the seminal papers of F. Black and M. Scholes [B-S 73] and R. Merton [M 73] - with the machinery of martingale theory. In particular it allows to reduce the pricing of a contingent claim to calculating expectation values, just in the way actuaries do for centuries. The (decisive) difference lies only in the fact that one does not take the expectation with respect to the original probabilty measure $P$ but with respect to an artificial "risk-neutral" probability measure $Q$, i.e. with respect to a measure under which the process $\left(S_{t}\right)_{t=0}^{N}$ is a martingale.

1980 Mathematics Subject Classification (1991 Revision). Primary 60 H 05;.

Key words and phrases. Equivalent Martingale Measure, Pricing by Arbitrage. 
The subtle point in the "Fundamental Theorem of Asset Pricing" is to give a precise meaning to the word "essentially". In the general case of infinite or continuous time this problem turns out to be very delicate and needs notions such as "no free lunch" or "no free lunch with bounded risk" generalizing the concept of "no arbitrage" in order to obtain saisfactory theorems (compare [H-K 79], [H-P 81], [K 81], [D-H 86], [St 90], [A-S 90],[F-S 90], [D 91], [S 92]).

But in the presently considered case of finite discrete time Dalang, Morton and Willinger showed that there is a nice and clear-cut theorem which may be phrased by using only the classical notion of "no arbitrage".

Let us give some precise definitions: $\left(\Omega, \mathcal{F},\left(\mathcal{F}_{t}\right)_{t=0}^{N}, P\right)$ will denote a filtered probability space and we assume that the process $\left(S_{t}\right)_{t=0}^{N}$ is adapted to the filtration $\left(\mathcal{F}_{t}\right)_{t=0}^{N}$.

A probability measure $Q$ on $\mathcal{F}$ will be called equivalent to $P$ if $Q$ and $P$ have the same null sets or - equivalently - if the mutual Radon-Nikodym derivatives exist.

We say that an equivalent probability measure $Q$ is an equivalent martingale measure for $\left(S_{t}\right)_{t=0}^{N}$ if $\left(S_{t}\right)_{t=0}^{N}$ is a martingale under $Q$, i.e., each $S_{t}$ is $Q$-integrable and for each $t=1, \ldots, N$, we have

$$
E_{Q}\left(\left(S_{t}-S_{t-1}\right) \mid \mathcal{F}_{t-1}\right) \equiv 0 .
$$

We say that the process $\left(S_{t}\right)_{t=0}^{N}$ satisfies the no arbitrage condition if for $t=$ $1, \ldots, N$ and each $\mathcal{F}_{t-1}$-measurable bounded $\mathbb{R}^{d}$-valued function $h$ such that

$$
\left(h(\omega), S_{t}(\omega)-S_{t-1}(\omega)\right) \geq 0 \quad P-\text { a.s. }
$$

we have

$$
\left(h(\omega), S_{t}(\omega)-S_{t-1}(\omega)\right)=0 \quad P-\text { a.s. }
$$

Here (.,.) denotes the inner product on $\mathbb{R}^{d}$.

The "no arbitrage condition" has a direct economic interpretation: It should not be possible to perform a trading operation on the stock price process $\left(S_{t}\right)_{t=0}^{N}$, described by the random variable $h$, such that the net result is almost surely nonnegative without being almost surely zero. It is reasonable to argue that a "good" model $\left(S_{t}\right)_{t=0}^{N}$ of a financial market should satisfy this assumption. The argument is that otherwise there would be economic agents taking advantage of this arbitrage opportunity which would quickly make it disappear.

It is almost obvious that the existence of an equivalent martingale measure $Q$ implies that the process $\left(S_{t}\right)_{t=0}^{N}$ satisfies the no arbitrage condition. Indeed, if $\left(S_{t}\right)_{t=0}^{N}$ is a martingale with respect to $Q$ then we have for each $\mathcal{F}_{t-1}$-measurable bounded $\mathbb{R}^{d}$-valued function $h$ that

$$
E_{Q}\left(h(\omega), S_{t}(\omega)-S_{t-1}(\omega)\right)=0 .
$$

If we have in addition that $\left(h(\omega), S_{t}(\omega)-S_{t-1}(\omega)\right) \geq 0 P$-almost surely (and therefore $Q$-almost surely) we conclude that $\left(h(\omega), S_{t}(\omega)-S_{t-1}(\omega)\right)=0 Q$-almost surely (and therefore $P$-almost surely). This shows that the process $\left(S_{t}\right)_{t=0}^{N}$ satisfies the no arbitrage condition.

The point of the Dalang-Morton-Willinger theorem lies in the fact that the reverse implication also holds true: 
1.1 Theorem (Dalang, Morton and Willinger). An adapted $\mathbb{R}^{d}$-valued process $\left(S_{t}\right)_{t=0}^{N}$ satisfies the no arbitrage condition if and only if there exists an equivalent martingale measure.

In this case the equivalent martingale measure $Q$ may be chosen such that the density $d Q / d P$ is uniformly bounded.

Some comments on this theorem are in order: Of course, the theorem applies in particular to the case $d=1$, i.e., the classical case where only one stock is considered. In this case the theorem was obtained by Back and Pliska [B-P 90] - who also conjectured the theorem for general $d \in \mathbb{N}$ - and the proof is substantially easier (compare remark 2.8 below). The case $d>1$ is much more delicate and needs some kind of geometric argument. We have dealt with these difficulties by using orthogonality arguments in properly chosen spaces.

One should note that the no arbitrage condition imposes no integrability assumptions on the process $\left(S_{t}\right)_{t=0}^{N}$ (compare [D-M-W 90] for a discussion of this remarkable feature of the theorem).

The theorem breaks down in the present form if one passes to infinite time (see [D-M-W 90] remark 2.7 for an easy counterexample) and it also breaks down if one passes to infinitly many stocks (see 2.8 below; compare also [A-H 92] for the relevance of considering the case of infinitly many stocks).

Let us now give an outline of the paper: Section 2 is devoted to the proof of theorem 1.1. We shall see that the essential step is contained in the key lemma 2.1 which in turn is similar to a lemma of C. Stricker [St 90]. Finally in section 3 we present some wellknown results used in section 2 and provide proofs for them to keep the paper entirely selfcontained.

To end the introductory section we shall reconsider the notion of no arbitrage and cast it into more mathematical terms. By $L^{0}\left(\Omega, \mathcal{F}, P ; \mathbb{R}^{d}\right)$ we denote the space of all (equivalence classes of) $\mathbb{R}^{d}$-valued $\mathcal{F}$-measurable random variables, which is a complete topological vector space if equipped with the topology of convergence in measure.

Denote by $K$ the subspace of $L^{0}(\Omega, \mathcal{F}, P)$ formed by the stochastic integrals on the process $\left(S_{t}\right)_{t=0}^{N}$, i.e.,

$$
K=\left\{\sum_{i=1}^{N}\left(S_{i}(\omega)-S_{i-1}(\omega), h_{i}(\omega)\right): h_{i} \in L^{0}\left(\Omega, \mathcal{F}_{i-1}, P ; \mathbb{R}^{d}\right) \text { for } 1 \leq i \leq N\right\}
$$

1.2 Lemma. The process $\left(S_{t}\right)_{t=0}^{N}$ satisfies the no arbitrage condition if and only
if

$$
K \cap L_{+}^{0}(\Omega, \mathcal{F}, P)=\{0\}
$$

Proof. As the functions $\left(h(\omega), S_{t}(\omega)-S_{t-1}(\omega)\right)$ appearing in the definition of the no arbitrage condition are elements of $K$ it is clear $K \cap L_{+}^{0}=\{0\}$ implies the no arbitrage condition. 
Conversely we argue by induction on $N$. If $N=1$ we deduce the validity of $K \cap L_{+}^{0}=\{0\}$ from the no arbitrage condition by simply observing that it makes no difference to assume that the function $h$ appearing in the definition of the no arbitrage condition is assumed to be bounded or not.

Now suppose that the lemma holds true for $N-1$ and consider a function

$$
f_{N}=\sum_{i=1}^{N}\left(h_{i}(\omega), S_{i}(\omega)-S_{i-1}(\omega)\right)
$$

where $h_{i} \in L^{0}\left(\Omega, \mathcal{F}_{i-1}, P ; \mathbb{R}^{d}\right)$ for $1 \leq i \leq N$ and suppose that $f_{N} \geq 0$. We have to show that $f_{N} \equiv 0$. To do so consider

$$
f_{N-1}=\sum_{i=1}^{N-1}\left(h_{i}(\omega), S_{i}(\omega)-S_{i-1}(\omega)\right)
$$

If $f_{N-1} \equiv 0$ the assertion $f_{N} \equiv 0$ follows from the above considered case $N=1$. If $f_{N-1} \not \equiv 0$ then by our inductive hypothesis $A=\left\{f_{N-1}<0\right\}$ is an element of $\mathcal{F}_{N-1}$ with strictly positive measure. Hence

$$
f=\left(h_{N}(\omega), S_{N}(\omega)-S_{N-1}(\omega)\right) \cdot \chi_{A}
$$

satisfies $f \geq 0$ and $f \not \equiv 0$ which again gives a contradiction to the case $N=1$ thus finishing the proof.

\section{The Proof of the Theorem}

We shall isolate the technical content of theorem 1.1 in the subsequent lemma 2.1. This result will quickly imply the theorem (see 2.2 below).

2.1 Key Lemma. Let $\left(\Omega, \mathcal{F}_{1}, P\right)$ be a probability space, $Y$ an $\mathcal{F}_{1}$-measurable $\mathbb{R}^{d}$-valued function, i.e., $Y \in L^{0}\left(\Omega, \mathcal{F}_{1}, P ; \mathbb{R}^{d}\right)$ and $\mathcal{F}_{0}$ a sub- $\sigma$-algebra of $\mathcal{F}_{1}$.

Similarly as above denote by $K$ the subspace of $L^{0}\left(\Omega, \mathcal{F}_{1}, P\right)$

$$
K=\left\{(h, Y): h \in L^{0}\left(\Omega, \mathcal{F}_{0}, P ; \mathbb{R}^{d}\right)\right\}
$$

and by $K-L_{+}^{0}$ the convex cone

$$
K-L_{+}^{0}\left(\Omega, \mathcal{F}_{1}, P\right)=\left\{f=(h, Y)-g: h \in L^{0}\left(\Omega, \mathcal{F}_{0}, P ; \mathbb{R}^{d}\right), g \in L_{+}^{0}\left(\Omega, \mathcal{F}_{1}, P\right)\right\}
$$

If $K \cap L_{+}^{0}\left(\Omega, \mathcal{F}_{1}, P\right)=\{0\}$ then $K-L_{+}^{0}\left(\Omega, \mathcal{F}_{1}, P\right)$ is closed in $L^{0}\left(\Omega, \mathcal{F}_{1}, P\right)$ with respect to the topology of convergence in measure.

Let us show how the key lemma implies theorem 1.1. 
2.2 Proof of theorem 1.1. Assuming that the no arbitrage condition holds true we have to construct a martingale measure $Q$ with bounded density function.

We first show the theorem in the case $N=1$, i.e., for the cases of of a two period model. Let $\left(S_{0}, S_{1}\right)$ be two $\mathbb{R}^{d}$-valued random variables adapted to the pair of $\sigma$ algebras $\left(\mathcal{F}_{0}, \mathcal{F}_{1}\right)$. Let $Y(\omega)=\left(S_{1}(\omega)-S_{0}(\omega)\right) / w(\omega)$ where $w$ is the weight function defined by

$$
w(\omega)=\max \left(\left\|S_{0}(\omega)\right\|_{\mathbb{R}^{d}},\left\|S_{1}(\omega)\right\|_{\mathbb{R}^{d}}, 1\right) .
$$

Using the notation of lemma 2.1 we have that $K \cap L_{+}^{0}\left(\Omega, \mathcal{F}_{1}, P\right)=\{0\}$ iff $\left(S_{0}, S_{1}\right)$ satisfies the no-arbitrage condition (NA) (lemma 1.2). Admitting the key lemma we deduce that this implies that $K-L_{+}^{0}$ is closed in $L^{0}\left(\Omega, \mathcal{F}_{1}, P\right)$ whence a fortiori $\left(K-L_{+}^{0}\right) \cap L^{1}\left(\Omega, \mathcal{F}_{1}, P\right)$ is closed in the space $L^{1}\left(\Omega, \mathcal{F}_{1}, P\right)$ with respect to the $L^{1}$ norm and disjoint from $L_{+}^{1}\left(\Omega, \mathcal{F}_{1}, P\right) \backslash\{0\}$. By Yan's theorem (see 3.1 below) there is $g \in L^{\infty}\left(\Omega, \mathcal{F}_{1}, P\right), g(\omega)>0$ almost surely such that $g$ - regarded as a functional on $L^{1}\left(\Omega, \mathcal{F}_{1}, P\right)$ - is less than or equal to 0 on the convex cone $\left(K-L_{+}^{0}\right) \cap L^{1}$. In particular for each $h \in L^{\infty}\left(\Omega, \mathcal{F}_{0}, P ; \mathbb{R}^{d}\right)$ we have

$$
\begin{gathered}
\int(h(\omega), Y(\omega)) \cdot g(\omega) d P(\omega)= \\
=\int\left(h(\omega), S_{1}(\omega)-S_{0}(\omega)\right) \cdot(g(\omega) / w(\omega)) d P(\omega) \leq 0 .
\end{gathered}
$$

By repeating the argument with $h$ replaced by $-h$ we deduce that equality holds true above. Let $Q$ be the measure on $\mathcal{F}_{1}$ with density function $c \cdot g(\omega) / w(\omega)$ where the normalizing factor $c \in \mathbb{R}_{+}$is chosen such that $Q(\Omega)=1$. Then $Q$ is a probability measure equivalent to $P$ and for each $h \in L^{\infty}\left(\Omega, \mathcal{F}_{0}, P, \mathbb{R}^{d}\right)$ the function $\left(h(\omega), S_{1}(\omega)-S_{0}(\omega)\right)$ is $Q$-integrable with $Q$-integral equal to 0 . In other words $\left(S_{1}, S_{0}\right)$ is a martingale with respect to $Q$ and the filtration $\left(\mathcal{F}_{0}, \mathcal{F}_{1}\right)$.

We now pass to the general case $N \in \mathbb{N}$ and proceed by induction on $N$ : Suppose that the theorem holds true for all processes of length $N-1$. Given the process $\left(S_{t}\right)_{t=0}^{N}$ apply the induction hypothesis to $\left(S_{t}\right)_{t=1}^{N}$ to obtain a bounded function $d_{N}$ such that the probability measure $Q_{N}$ on $\mathcal{F}$ with density function $d Q_{N} / d P=d_{N}$ is equivalent to $P$ and turns $\left(S_{t}\right)_{t=1}^{N}$ into a martingale (with respect to the filtration $\left.\left(\mathcal{F}_{t}\right)_{t=1}^{N}\right)$.

Observe that for any $\mathcal{F}_{1}$-measurable function $d_{1}$ such that $d_{1}>0$ almost surely and satisfying the normalising condition $E_{P}\left(d_{1} \cdot d_{N}\right)=1$ the measure $Q$ with density $d Q / d P=d_{1} \cdot d_{N}$ still is an equivalent martingale measure for $\left(S_{t}\right)_{t=1}^{N}$.

Now apply the first step to the process $\left(S_{t}\right)_{t=0}^{1}$ relative to $\left(\Omega, \mathcal{F}_{1},\left(\mathcal{F}_{t}\right)_{t=0}^{1},\left.Q_{N}\right|_{\mathcal{F}_{1}}\right)$ to obtain a bounded $\mathcal{F}_{1}$-measurable function $d_{1}$ such that the measure $Q_{1}$ on $\mathcal{F}_{1}$ with density $d Q_{1} / d Q_{N}=d_{1}$ turns $\left(S_{t}\right)_{t=0}^{1}$ into a martingale.

The measure $Q$ on $\mathcal{F}$ with density $\frac{d Q}{d P}=\frac{d Q}{d Q_{N}} \cdot \frac{d Q_{N}}{d P}=d_{1} \cdot d_{N}$ now turns the whole process $\left(S_{t}\right)_{t=0}^{N}$ into a martingale, thus finishing the proof.

Let us now turn to the proof of lemma 2.1. This result should be compared to the following result of C. Stricker ([St 90] lemma 2) which asserts that — in the setting 
of lemma 2.1 above - the linear space $K$ is closed in $L^{0}$. For the convinience of the reader and in view of an irritating misprint in ([St 90] lemma 2) we restate this result and prove it (3.4 below).

2.3 Stricker's Lemma [St 90]. Let again $\left(\Omega, \mathcal{F}_{1}, P\right)$ be a probability space, $Y \in L^{0}\left(\Omega, \mathcal{F}_{1}, P ; \mathbb{R}^{d}\right), \mathcal{F}_{0}$ a sub- $\sigma$-algebra of $\mathcal{F}_{1}$ and denote by $K$ the subspace of $L^{0}\left(\Omega, \mathcal{F}_{1}, P\right)$

$$
K=\left\{(h, Y): h \in L^{0}\left(\Omega, \mathcal{F}_{0}, P ; \mathbb{R}^{d}\right)\right\}
$$

Then $K$ is closed in $L^{0}\left(\Omega, \mathcal{F}_{1}, P\right)$.

Note that in Stricker's lemma it is not necessary to make the assumption $K \cap L_{+}^{0}=$ $\{0\}$.

To see why this assumption is indeed necessary in lemma 2.1 to conclude that the convex cone $K-L_{+}^{0}$ is closed consider the following easy example: Let $\left(\Omega, \mathcal{F}_{1}, P\right)$ be $[0,1]$ equipped with the Lebesgue $\sigma$-algebra $\mathcal{F}_{1}$ and Lebesgue measure $P$. Let $\mathcal{F}_{0}$ be the trivial $\sigma$-algebra and $Y$ the identity function on $[0,1]$. It is instructive to verify that the closure of $K-L_{+}^{0}$ equals the whole space $L^{0}\left(\Omega, \mathcal{F}_{1}, P\right)$ while the function $f \equiv 1$ does not belong to $K-L_{+}^{0}$. Hence $K-L_{+}^{0}$ is not closed in this case.

Note that in this example the function $Y$ takes only non-negative values. To rule out the phenomenon occuring in this example we need the assumption $K \cap L_{+}^{0}=\{0\}$ in lemma 2.1.

After this motivating example we now start the proof of lemma 2.1. The first step 2.4 is an easy reduction. Note that in the proof of theorem 1.1 we applied lemma 2.1 only to a bounded function $Y$. Hence a reader interested only in the proof of this theorem may completely omit the next lemma.

2.4. Lemma. If lemma 2.1 holds true for each $Y \in L^{\infty}\left(\Omega, \mathcal{F}_{1}, P ; \mathbb{R}^{d}\right)$ then it holds true for each $Y \in L^{0}\left(\Omega, \mathcal{F}_{1}, P ; \mathbb{R}^{d}\right)$. In other words there is no loss of generality in assuming $Y$ to be bounded in proving lemma 2.1 .

Proof. Let $Y \in L^{0}\left(\Omega, \mathcal{F}_{1}, P ; \mathbb{R}^{d}\right)$ and define

$$
\varphi(\omega)=\min \left(\|Y(\omega)\|^{-1}, 1\right) .
$$

Note that the multiplication operator

$$
\begin{aligned}
M_{\varphi}: L^{0}\left(\Omega, \mathcal{F}_{1}, P\right) & \mapsto L^{0}\left(\Omega, \mathcal{F}_{1}, P\right) \\
M_{\varphi}(f)(\omega) & =\varphi(\omega) \cdot f(\omega)
\end{aligned}
$$

defines an orderpreserving isomorphism on the topological vector space $L^{0}\left(\Omega, \mathcal{F}_{1}, P\right)$.

Hence $M_{\varphi}(K) \cap L_{+}^{0}=\{0\}$ iff $K \cap L_{+}^{0}=\{0\}$ and $K-L_{+}^{0}\left(\Omega, \mathcal{F}_{1}, P\right)$ is closed iff $M_{\varphi}\left(K-L_{+}^{0}\right)=M_{\varphi}(K)-L_{+}^{0}$ is closed. But

$$
M_{\varphi}(K)=\left\{(h(\omega), \varphi(\omega) \cdot Y(\omega)): h \in L^{0}\left(\Omega, \mathcal{F}_{0}, P ; \mathbb{R}^{d}\right)\right\}
$$

and $\tilde{Y}(\omega)=\varphi(\omega) \cdot Y(\omega)$ is in $L^{\infty}\left(\Omega, \mathcal{F}_{1}, P ; \mathbb{R}^{d}\right)$. 
Let us fix from now on $Y \in L^{\infty}\left(\Omega, \mathcal{F}_{1}, P ; \mathbb{R}^{d}\right)$. We define the following subspaces of $L^{0}$ :

$$
\begin{aligned}
N & =\left\{k \in L^{0}\left(\Omega, \mathcal{F}_{0}, P ; \mathbb{R}^{d}\right):(k(\omega), Y(\omega))=0 P-\text { a.s. }\right\} \\
N^{\perp} & =\left\{h \in L^{0}\left(\Omega, \mathcal{F}_{0}, P ; \mathbb{R}^{d}\right):(k(\omega), h(\omega))=0 P-\text { a.s. for each } k \in N\right\}
\end{aligned}
$$

Intuitively $N$ and $N^{\perp}$ form an "orthogonal" decomposition of the space $L^{0}$ (which is, of course, not a welldefined statement). What we mean by that is made precise in the next statement.

2.5 Lemma. There is a continuous surjective projection

$$
\pi: L^{0}\left(\Omega, \mathcal{F}_{0}, P ; \mathbb{R}^{d}\right) \rightarrow N^{\perp}
$$

with $\operatorname{ker}(\pi)=N$. In other words

$$
L^{0}\left(\Omega, \mathcal{F}_{0}, P ; \mathbb{R}^{d}\right)=N \oplus N^{\perp}
$$

We then have, for each $h \in L^{0}\left(\Omega, \mathcal{F}_{0}, P ; \mathbb{R}^{d}\right)$,

$$
(h(\omega), Y(\omega))=(\pi(h)(\omega), Y(\omega)) \quad P \text {-a.s. }
$$

Proof. Denote by $H$ the Hilbert space $L^{2}\left(\Omega, \mathcal{F}_{0}, P ; \mathbb{R}^{d}\right)$ equipped with its usual inner product $\langle\cdot, \cdot\rangle$. Clearly $N \cap H$ and $N^{\perp} \cap H$ are orthogonal subspaces of $H$.

To prove that $N \cap H$ and $N^{\perp} \cap H$ span $H$ suppose to the contrary that there is $h \in H, h$ orthogonal to $N \cap H$ and $h \notin N^{\perp} \cap H$. Then there is $k \in N$ such that $(k(\omega), h(\omega))$ does not vanish a.s. By changing sign if necessary the set

$$
A=\{(k(\omega), h(\omega))>0\}
$$

is an element of $\mathcal{F}_{0}$ with positive $P$-measure. Letting

$$
\tilde{k}(\omega)=\chi_{A}(\omega) \cdot k(\omega) /\|k(\omega)\|
$$

we have found an element of $N \cap H$ for which

$$
<\tilde{k}, h>=E((\tilde{k}(\omega), h(\omega))>0
$$

a contradiction showing that

$$
H=(N \cap H) \oplus\left(N^{\perp} \cap H\right) .
$$

Denote by $p: H \mapsto N^{\perp} \cap H$ the orthogonal projection. To extend $p$ to the space $L^{0}\left(\Omega, \mathcal{F}_{0}, P ; \mathbb{R}^{d}\right)$ note that $N$ and $N^{\perp}$ are closed under multiplication with scalarvalued $\mathcal{F}_{0}$-measurable functions. Hence we may unambigously define

$$
\pi: L^{0}\left(\Omega, \mathcal{F}_{0}, P ; \mathbb{R}^{d}\right) \rightarrow N^{\perp}
$$




$$
\pi(f)(\omega)=\|f(\omega)\| \cdot \pi(f(\omega) /\|f(\omega)\|)(\omega)
$$

with the understanding $f(\omega) /\|f(\omega)\|=0$ if $f(\omega)=0$. Clearly $\pi$ has the properties stated in the lemma.

We now have decomposed $L^{0}\left(\Omega, \mathcal{F}_{0}, P ; \mathbb{R}^{d}\right)$ into the space $N$ "strongly orthogonal" to $Y$ and its "orthogonal complement" $N^{\perp}$. Similarly we decompose $\Omega$ into the elements $\Omega_{N}$ and $\Omega_{N}^{c}$ of $\mathcal{F}_{0}$

$$
\Omega_{N}=\left\{E\left(\|Y(\omega)\| \mid \mathcal{F}_{0}\right)=0\right\} \quad \text { and } \quad \Omega_{N}^{c}=\Omega \backslash \Omega_{N}=\left\{E\left(\|Y(\omega)\| \mid \mathcal{F}_{0}\right)>0\right\} .
$$

For the rest of this section we shall assume that $K \cap L_{+}^{0}=\{0\}$ holds true.

2.6 Lemma. For $A \in \mathcal{F}_{0}, A \subseteq \Omega_{N}^{c}$ define

$$
\mu(A)=\inf \left\{E\left((h(\omega), Y(\omega))_{+}\right): h \in N^{\perp},\|h(\omega)\|=1 \text { for } \omega \in A\right\}
$$

Then $\mu$ is well defined and $P(A)>0$ implies $\mu(A)>0$.

Proof. To show that the definition of $\mu$ makes sense we shall show that there is $h \in N^{\perp}$ such that $\|h(\omega)\|=1$ almost surely on $\Omega_{N}^{c}$.

For $i=1, \ldots, d$ define $h_{i} \in N^{\perp}$ by

$$
h_{i}(\omega)=\pi\left(e_{i} \cdot 1\right)
$$

where $e_{i}$ denotes the $i^{\prime}$ th unit vector of $\mathbb{R}^{d}$ and 1 the constant function 1 .

Let $S_{i} \in \mathcal{F}_{0}$ denote the support of $h_{i}$ and $T_{i} \in \mathcal{F}_{0}$ denote the support of $E\left(\left|Y_{i}\right| \mid\right.$ $\mathcal{F}_{0}$ ), where $Y_{i}$ denotes the $i^{\prime}$ th coordinate of $Y$. Clearly $\bigcup_{i=1}^{d} T_{i}=\Omega_{N}^{c}$ and we shall show that $S_{i}=T_{i}$ for $1 \leq i \leq d$ (in the sense that the symmetric difference $S_{i} \triangle T_{i}$ is a null-set). This will imply that

$$
h=\sum_{i=1}^{d}\left(h_{i}(\omega) /\left\|h_{i}(\omega)\right\|\right) \cdot \chi_{\left(S_{i} \backslash \bigcup_{j<i} S_{j}\right)}
$$

is an element of $N^{\perp}$ such that $\|h(\omega)\|=1$ a.s. on $\Omega_{F}$.

To verify that $S_{i} \subseteq T_{i}$ note that $e_{i} \chi_{\Omega \backslash T_{i}}$ is an element of $N$ which clearly implies that the support of $h_{i}$ is disjoint from $\Omega \backslash T_{i}$.

To see that $S_{i} \supseteq T_{i}$ suppose to the contrary that $P\left(T_{i} \backslash S_{i}\right)>0$. Then $e_{i} \chi_{T_{i} \backslash S_{i}}$ is an element of $N$ as $\pi$ maps it to zero. By definition $\left(e_{i} \chi_{T_{i} \backslash S_{i}}, Y(\omega)\right)=\chi_{T_{i} \backslash S_{i}} Y_{i}(\omega)$ vanishes a.s. contradicting the fact that

$$
E\left(\chi_{T_{i} \backslash S_{i}}|Y|\right)=E\left(\chi_{T_{i} \backslash S_{i}} E\left(\left|Y_{i}\right| \mid \mathcal{F}_{0}\right)\right)>0
$$

Hence we have shown that $\mu$ is well defined. 
We now turn to the proof of the fact that $\mu(A)>0$ for $A \in \mathcal{F}_{0}, A \subseteq \Omega_{N}^{c}$, $P(A)>0$. Suppose to the contrary that there is a sequence $\left(h_{n}\right)_{n=1}^{\infty} \in N^{\perp}$ supported by $A,\left\|h_{n}(\omega)\right\|=1$ for $\omega \in A$ such that

$$
\lim _{n \rightarrow \infty} E\left(\left(h_{n}(\omega), Y(\omega)\right)_{+}\right)=0
$$

We claim that this implies

$$
\lim _{n \rightarrow \infty} E\left(\left(h_{n}(\omega), Y(\omega)\right)_{-}\right)=0 \text {. }
$$

Indeed, otherwise we may find - by passing to a subsequence - some $\alpha>0$ such that

$$
E\left(\left(h_{n}(\omega), Y(\omega)\right)_{-}\right) \geq \alpha .
$$

By lemma 3.2 below we may find a sequence of convex combinations of $\left(h_{n}\right)_{n=1}^{\infty}$ - still denoted by $\left(h_{n}\right)_{n=1}^{\infty}$ - such that $\left(h_{n}\right)_{n=1}^{\infty}$ converges a.s. to some $h_{0} \in N^{\perp}$ for which we get by Lebesgue's theorem (using the boundedness of $\left(h_{n}\right)_{n=1}^{\infty}$ and $Y$ ) that

$$
E\left(\left(h_{0}(\omega), Y(\omega)\right)_{+}\right)=0 \quad \text { while } \quad E\left(\left(h_{0}(\omega), Y(\omega)\right)_{-}\right) \geq \alpha>0 .
$$

Hence $-\left(h_{0}(\omega), Y(\omega)\right)$ is in $\left(L_{+}^{0} \backslash\{0\}\right) \cap K$, a contradiction proving (2).

Equalities (1) and (2) imply that $\left(h_{n}(\omega), Y(\omega)\right)_{n=1}^{\infty}$ tends to zero in mean, hence - by passing to a subsequence - almost surely.

On the other hand lemma 3.3 implies that there is a bounded sequence $g_{n} \in$ $L^{\infty}\left(\Omega, \mathcal{F}_{0}, P\right)$ and a sequence $f_{n} \in \operatorname{conv}\left(g_{n} h_{n}, g_{n+1} h_{n+1}, \ldots\right)$ such that $f_{n}$ converges a.s. to some $f_{0} \not \equiv 0$.

This gives the desired contradiction proving that $\mu(A)>0$ : On one hand side $f_{0} \in N^{\perp}$ as each $g_{n} h_{n}$ is in $N^{\perp}$; on the other hand $f_{0} \in N$ as $\left(f_{0}(\omega), Y(\omega)\right)=0$ almost surely.

2.7. Lemma. Let $\left(h_{n}\right)_{n=1}^{\infty}$ be a sequence in $N^{\perp}$ such that

$$
\Psi(\omega)=\sup _{n}\left(h_{n}(\omega), Y(\omega)\right)_{+}
$$

is finite almost surely. Then

$$
\Phi(\omega)=\sup _{n}\left\|h_{n}(\omega)\right\|
$$

is finite almost surely too.

Proof. Suppose to the contrary that for $A=\{\Phi(\omega)=+\infty\}$ we have that $P(A)>$ 0 . For $n, m \in \mathbb{N}$, let

$$
A_{n, m}=A \cap\left\{\left\|h_{m}\right\| \geq n\right\} .
$$

By our assumption we have, for each $n \in \mathbb{N}$,

$$
\bigcup_{m=n}^{\infty} A_{n, m}=A .
$$


Define, for $n \in \mathbb{N}$

$$
\tilde{h}_{n}=\sum_{m=n}^{\infty}\left(h_{m} /\left\|h_{m}\right\|\right) \chi_{A_{n, m} \backslash \bigcup_{j=n}^{m-1} A_{n, j}}
$$

to obtain a sequence $\left(\tilde{h}_{n}\right)_{n=1}^{\infty}$ in $N^{\perp}$ supported by $A$ such that $\left\|\tilde{h}_{n}(\omega)\right\|=1$ for almost all $\omega \in A$ and such that

$$
\lim _{n \rightarrow \infty}\left(\left(\tilde{h}_{n}(\omega), Y(\omega)\right)_{+}\right)=0 \quad P \text {-a.s. }
$$

Hence by Lebesgue's theorem

$$
\lim _{n \rightarrow \infty} E\left(\left(\tilde{h}_{n}, Y\right)_{+}\right)=0
$$

contradicting lemma 2.6

We now have assembled all the ingredients for the proof of the key lemma.

Proof of lemma 2.1. We have to show that $K-L_{+}^{0}\left(\Omega, \mathcal{F}_{1}, P\right)$ is closed.

Let $f \in L^{0}\left(\Omega, \mathcal{F}_{1}, P\right),\left(h_{n}\right)_{n=1}^{\infty} \in L^{0}\left(\Omega, \mathcal{F}_{0}, P ; \mathbb{R}^{d}\right)$ and $\left(k_{n}\right)_{n=1}^{\infty} \in L_{+}^{0}\left(\Omega, \mathcal{F}_{1}, P\right)$ be such that

$$
\lim _{n \rightarrow \infty}\left(\left(h_{n}(\omega), Y(\omega)\right)-k_{n}(\omega)\right)=f(\omega)
$$

the limit taken with respect to convergence in measure. We have to show that $f$ is contained in $K-L_{+}^{0}\left(\Omega, \mathcal{F}_{1}, P\right)$, i.e. that there is $h \in L^{0}\left(\Omega, \mathcal{F}_{0}, P\right)$ such that

$$
(h(\omega), Y(\omega)) \geq f(\omega) \quad P \text {-a.s. }
$$

By passing to a subsequence we may assume that the above sequence converges almost surely and by lemma 2.5 we may suppose - by passing from $h_{n}$ to $\pi\left(h_{n}\right)$ that $h_{n} \in N^{\perp}$. Note that

$$
\sup _{n}\left(-h_{n}(\omega), Y(\omega)\right)<+\infty \quad P \text {-a.s. }
$$

whence by lemma 2.6

$$
\Phi(\omega)=\sup _{n}\left\|h_{n}(\omega)\right\|<+\infty \quad P \text {-a.s. }
$$

By lemma 3.2 there is a sequence of convex combinations $f_{n} \in \operatorname{conv}\left(h_{n}, h_{n+1}, \ldots\right)$ converging a.s. to some $h \in L^{0}\left(\Omega, \mathcal{F}_{0}, P ; \mathbb{R}^{d}\right)$. As

$$
(h(\omega), Y(\omega)) \geq f(\omega) \quad P \text {-a.s. }
$$

we have completed the proof of the key lemma 2.1. 
2.8. Remark. It may be instructive to check the above arguments in the (easier) case $d=1$ : Using the notation of the above lemmata we then have that $N^{\perp}$ (resp. $N)$ is the subspace of $L^{0}\left(\Omega, \mathcal{F}_{0}, P\right)$ of functions supported by $\Omega_{N}^{c}$ (resp. $\left.\Omega_{N}\right)$ and the projection $\pi$ is simply multiplication by $\chi_{\Omega_{N}^{c}}$. The set function $\mu$ on $\left.\mathcal{F}_{0}\right|_{\Omega_{N}^{c}}$ is just the measure whose density is given, for $\omega \in \Omega_{N}^{c}$, by

$$
(d \mu / d P)(\omega)=\min \left(E\left(Y_{+} \mid \mathcal{F}_{0}\right), E\left(Y_{-} \mid \mathcal{F}_{0}\right)\right)
$$

In the case $d \geq 2$ there is no such simple interpretation. The reader interested in getting additional motivation might compare the above developed notions with the geometric concepts used in [D-M-W 90].

Finally we give a very easy example showing that theorem 1.1 breaks down in the case of infinitly many securities, i.e., $d=\infty$. Consider the space $\mathbb{R}^{\mathbb{N}}$ of all sequences of real numbers which is in natural duality with the space $\mathbb{R}^{(\mathbb{N})}$ of sequences of real numbers with finite support. Let $\Omega=\mathbb{N}, \mathcal{F}_{0}=\{\Phi, \Omega\}, \mathcal{F}=\mathcal{F}_{1}$ the power set of $\mathbb{N}$ and $P=\sum_{n=1}^{\infty} 2^{-n} \delta_{n}$. Let the $\mathbb{R}^{\mathbb{N}}$-valued process $\left(S_{t}\right)_{t=0}^{1}$ be given by $S_{0} \equiv 0$ and the $j$-th coordinate of $S_{1}$ given by

$$
S_{1}^{j}(\omega)=\left\{\begin{array}{cl}
1, & \text { for } \omega=j \\
-1, & \text { for } \omega=j+1 \\
0, & \text { elsewhere }
\end{array}\right.
$$

Clearly there is no equivalent martingale measure $Q$ on $\mathcal{F}$ turning $\left(S_{t}\right)_{t=0}^{1}$ into a martingale as such a measure $Q$ would have to verify $Q(j)=Q(j+1)$ for all $j \in \mathbb{N}$, which is clearly absurd.

On the other hand it is just as obvious that for every $\mathbb{R}^{(\mathbb{N})}$-valued $\mathcal{F}_{0}$-measurable (i.e., constant) function $h$ we have that the function

$$
\begin{aligned}
f(\omega) & =\left\langle h(\omega), S_{1}(\omega)-S_{0}(\omega)\right\rangle \\
& =\sum_{j=1}^{\infty} h^{j}(\omega) \cdot S_{1}^{j}(\omega)
\end{aligned}
$$

is nonnegative iff it is identically zero, which shows that $S$ satisfies the no arbitrage condition.

\section{Some wellknown results}

In this section we recall some results which we have used in the above proof. For the convenience of the reader we provide proofs for them.

The first result is a fundamental tool in the theory of semimartingales. It is due to Yan [Y 80] but a similar result has been obtained independently by Kreps [K 81] in the context of financial mathematics (compare [St 90], [A-S 90], [L 92], [S 92] [K 92] for other versions of this teorem). We formulate an easy version appropriate for our present setting. 
3.1 Theorem (Yan). Let $C$ be a closed convex cone in $L^{1}(P)$ containing $L_{-}^{1}$ and such that $C \cap L_{+}^{1}=\{0\}$. Then there is $g \in L^{\infty}$ with $g(\omega)>0$ for almost all $\omega \in \Omega$ and $\left.g\right|_{C} \leq 0$.

Proof. The proof is a combination of a Hahn-Banach and an exhaustion argument.

Step 1 (Hahn-Banach argument):

For each fixed $f \in L_{+}^{1}, f \neq 0$, there is $g \in L_{+}^{\infty}$ which is less than or equal to zero on $C$ such that

$$
\langle f, g\rangle=E(f g)>0 .
$$

Indeed, apply the separation theorem ( Schaefer (71), th. II, 9.2) to the closed convex set $C$ and the compact set $\{f\}$ to find $g \in L^{\infty}$ and $\alpha<\beta$ such that

$$
\left.g\right|_{C} \leq \alpha \text { and }\langle f, g\rangle>\beta .
$$

As $0 \in C$ we have $\alpha \geq 0$ and therefore $\langle f, g\rangle>0$. On the other hand $g$ is bounded from above on $C$ and therefore on $L_{-}^{1}$, i.e. $g \in L_{+}^{\infty}$. This proves step 1.

Step 2 (Exhaustion Argument):

Denote by $\mathcal{G}$ the set of all elements $g \in L_{+}^{\infty}, g$ being less than or equal to zero on $C$. As $0 \in \mathcal{G}$ (or by Step 1 ), $\mathcal{G}$ is nonempty.

Let $\mathcal{S}$ be the family of (equivalence classes of ) subsets of $\Omega$ formed by the supports of the elements $g \in \mathcal{G}$. Note that $\mathcal{S}$ is closed under countable unions, as for a sequence $\left(g_{n}\right)_{n=1}^{\infty} \in \mathcal{G}$ we may find strictly positive scalars $\left(\alpha_{n}\right)_{n=1}^{\infty}$, such that $\sum_{n=1}^{\infty} \alpha_{n} g_{n} \in \mathcal{G}$.

Hence there is $g_{0} \in \mathcal{G}$ such that for $S_{0}=\left\{g_{0}>0\right\}$ we have

$$
P\left(S_{0}\right)=\sup \{P(S): S \in \mathcal{S}\} .
$$

We shall show that $P\left(S_{0}\right)=1$ which readily shows that $g_{0}$ is strictly positive almost surely. If $P\left(S_{0}\right)<1$ then we could apply step 1 to $f=\chi_{\Omega \backslash S_{0}}$ to find $g_{1} \in \mathcal{G}$ with

$$
\left\langle f, g_{1}\right\rangle=\int_{\Omega \backslash S_{0}} g_{1}(\omega) d P(\omega)>0
$$

Hence $g_{0}+g_{1}$ would be an element of $\mathcal{G}$ whose support has $P$-measure strictly bigger than $P\left(S_{0}\right)$, a contradiction.

The next lemma is of folklore type and very useful. It may be viewed as a substitute of compactness, if one is ready to pass to convex combinations, which usually does not do much harm. Let us point out that the use of convex combinations allows for remarkable flexibility (compare [D-R-S 92]). Similarly as in Yan's theorem 3.1 above we only present an easy version and refer to [S 92] and [D-S 92] for more sophisticated versions of this result. 
3.2 Lemma. Let $\left(f_{n}\right)_{n=1}^{\infty}$ be a sequence in $L^{0}\left(\Omega, \mathcal{F}, P ; \mathbb{R}^{d}\right)$ with $\sup _{n}\left\|f_{n}(\omega)\right\|_{\mathbb{R}^{d}}$ almost surely finite. Then there exists a sequence $\left(g_{n}\right)_{n=1}^{\infty}$ of convex combinations $g_{n} \in \operatorname{conv}\left\{f_{n}, f_{n+1}, \ldots\right\}$ which converges almost surely.

Proof. First assume that the sequence $\left(f_{n}\right)_{n=1}^{\infty}$ is uniformly bounded with respect to the norm of $\mathbb{R}^{d}$. In particular this sequence is bounded in the Hilbert space $L^{2}\left(P ; \mathbb{R}^{d}\right)$ and by the reflexivity of Hilbert spaces there is a subsequence converging in the weak topology of $L^{2}\left(P ; \mathbb{R}^{d}\right)$. By the Hahn-Banach theorem the weak limit is in the norm closure of the convex hull of $\left(f_{j}\right)_{j=n}^{\infty}$, for each $n \in \mathbb{N}$, and therefore there is a sequence $g_{n} \in \operatorname{conv}\left\{f_{n}, f_{n+1}, \ldots\right\}$ which converges with respect to the norm of $L^{2}\left(P ; \mathbb{R}^{d}\right)$. Extracting once more a subsequence, if necessary, we may conclude that $\left(g_{n}\right)_{n=1}^{\infty}$ converges almost surely.

We now pass to the general case: Define the weight function

$$
w(\omega)=\sup _{n}\left\|f_{n}(\omega)\right\|_{\mathbb{R}^{d}}
$$

which by hypothesis is almost surely finite. Apply the first step to the uniformly bounded sequence $\left(f_{n} / w\right)_{n=1}^{\infty}$ to find a sequence of convex combinations that converges almost surely. But then obviously the same sequence of convex combinations taken from the sequence $\left(f_{n}\right)_{n=1}^{\infty}$ converges almost surely too.

Finally we prove a technical result which we needed in the proof of lemma 2.6 above. To get some motivation consider the sequence $\left(r_{n}\right)_{n=1}^{\infty}$ of Rademacher functions on $[0,1]$. It is instructive to apply lemma 3.2 to this situation: It is not hard to construct directly convex combinations $g_{n} \in \operatorname{conv}\left(r_{n}, r_{n+1}, \ldots\right)$ converging almost surely to some $g_{0}$. Obviously there are many ways to choose such sequences $\left(g_{n}\right)_{n=1}^{\infty}$ but one easily verifies that the limit function $g_{0}$ necessarily equals 0 .

In lemma 2.6 above we needed a limit different from zero and this is achieved by the following easy ad hoc argument:

3.3 Lemma. Let $\left(h_{n}\right)_{n=1}^{\infty}$ be a bounded sequence in $L^{2}\left(\Omega, \mathcal{F}, P ; \mathbb{R}^{d}\right)$ which stays bounded away from zero in probability, i.e., there is $\alpha>0$ such that $P\left\{\left\|h_{n}\right\| \geq \alpha\right\} \geq$ $\alpha$, for all $n \in \mathbb{N}$.

Then there is a bounded sequence $\left(g_{n}\right)_{n=1}^{\infty} \in L^{\infty}(\Omega, \mathcal{F}, P)$ and a sequence of convex combinations $f_{n} \in \operatorname{conv}\left(g_{n} h_{n}, g_{n+1} h_{n+1}, \ldots\right)$ such that $f_{n}$ converges almost surely to some $f_{0} \in L^{2}\left(\Omega, \mathcal{F}, P ; \mathbb{R}^{d}\right)$ with $f_{0} \not \equiv 0$.

Proof. Denoting by $h_{n}^{i}$ the i'th coordinate of $h_{n}$ we may find some $1 \leq i \leq d$ such that

$$
\limsup _{n \rightarrow \infty} E\left(\left|h_{n}^{i}\right|\right) \geq d^{-1} \alpha^{2}>0 .
$$

Let $g_{n}(\omega)=\operatorname{sign}\left(h_{n}^{i}(\omega)\right)$ and find a subsequence $\left(h_{n_{k}}\right)$ such that

$$
\lim _{k \rightarrow \infty} E\left(\left|h_{n_{k}}^{i}\right|\right)=\lim _{k \rightarrow \infty} E\left(g_{n_{k}} \cdot h_{n_{k}}^{i}\right)>0 .
$$

Finally apply the argument used in the first part of the proof of the preceding lemma 3.2 to choose a sequence $\left(f_{k}\right)_{k=1}^{\infty}$ such that $f_{k} \in \operatorname{conv}\left(g_{n_{k}} h_{n_{k}}, g_{n_{k+1}} h_{n_{k+1}}, \ldots\right)$ 
and such that $\left(f_{k}\right)_{k=1}^{\infty}$ converges a.s. to some $f_{0}$. As $\left(f_{k}\right)_{k=1}^{\infty}$ is uniformly integrable we infer from Vitali's theorem that $E\left(f_{0}^{i}\right)>0$, whence in particular that $f_{0} \not \equiv 0$.

To end this section we shall show how the concepts developed for the proof of lemma 2.1 also allow to give an easy proof of Stricker's lemma 2.3 above.

3.4 Proof of Stricker's lemma 2.3. Let $\left(h_{n}\right)_{n=1}^{\infty}$ be a sequence in $L^{0}\left(\Omega, \mathcal{F}_{0}, P ; \mathbb{R}^{d}\right)$ such that $\left(h_{n}(\omega), Y(\omega)\right)_{n=1}^{\infty}$ converges in measure. We have to show that there is $h_{0} \in L^{0}\left(\Omega, \mathcal{F}_{0}, P ; \mathbb{R}^{d}\right)$ such that $\left(h_{0}(\omega), Y(\omega)\right)=\lim _{n \rightarrow \infty}\left(h_{n}(\omega), Y(\omega)\right)$.

We claim that the sequence $\left(\pi\left(h_{n}\right)\right)_{n=1}^{\infty}$ converges in measure which will finish the proof in view of lemma 2.5 .

To prove the claim suppose to the contrary that there are sequences $\left(n_{j}\right)_{j=1}^{\infty}$ and $\left(m_{j}\right)_{j=1}^{\infty}$ tending to infinity such that the sequence $\left(k_{j}\right)_{j=1}^{\infty}=\left(\pi\left(h_{n_{j}}\right)-\pi\left(h_{m_{j}}\right)\right)_{j=1}^{\infty}$ stays bounded away from zero in measure. Note that the sequence

$$
\tilde{k}_{j}(\omega)=k_{j}(\omega) / \max \left(\left\|k_{j}(\omega)\right\|, 1\right)
$$

is a uniformly bounded sequence in $N^{\perp}$ staying bounded away from zero in measure and such that $\left(\tilde{k}_{j}, Y\right)_{j=1}^{\infty}$ tends to zero in measure and - by passing to a subsequence - almost surely.

Apply lemma 3.3 to find a uniformly bounded sequence $\left(g_{j}\right)_{j=1}^{\infty} \in L^{\infty}\left(\Omega, \mathcal{F}_{0}, P\right)$ and a sequence of convex combinations $f_{j} \in \operatorname{conv}\left(g_{j} \tilde{k}_{j}, g_{j+1} \tilde{k}_{j+1}, \ldots\right)$ such that $\left(f_{j}\right)_{j=1}^{\infty}$ converges almost surely to some $f_{0} \not \equiv 0$. Then $f_{0} \in N \cap N^{\perp}$ as $\left(f_{0}, Y\right) \equiv 0$, a contradiction finishing the proof. 


\section{References}

J. P. Ansel, C. Stricker (1990), Quelques remarques sur un théorème de Yan, Sém. de Probabilités, Springer Lecture Notes in Mathematics XXIV, 226-274.

Ph. Artzner, D. Heath (1992), Aproximate Completeness with Multiple Martingale Measures, preprint (17 p.).

K. Back, S. R. Pliska (1990), On the Fundamental Theorem of Asset Pricing with an infinite State Space, Journal of Mathematical Economics 20, 1 - 18.

F. Black, M. Scholes (1973), The Pricing of Options and Corporate Liabilities, J. of Political Economy 81, 637-654.

R. C. Dalang, A. Morton, W. Willinger (1990), Equivalent Martingale measures and no-arbitrage in stochastic securities market models, Stochastics and Stochastic Reports 29, 185-201.

F. Delbaen (1991), Representing Martingale Measures when Asset Prices are continuous and bounded, Math. Finance 2, 107 - 130.

F. Delbaen, W. Schachermayer (1992), A General Version of the Fundamental Theorem of Asset Pricing, in preparation.

J. Diestel, W. Ruess, W. Schachermayer (1992), On weak Compactness in $L^{1}(\mu, X)$, to appear in Proc. Am. Math. Soc..

D. Duffie, C. F. Huang (1986), Multiperiod security markets with differential information, J. Math. Econ. 15, 283-303.

P. Dybvig, S. Ross (1987), Arbitrage, in J. Eatwell, M. Milgate and P. Newmanm eds., The new Palgrave: A dictionary of Economics 1, McMillan London, 100 - 106.

H; Föllmer, M: Schweizer (1990), Hedging of Contingent Claims under Incomplete Information, mimeo, $22 \mathrm{p}$.

M. J. Harrison, D. M. Kreps (1979), Martingales and arbitrage in multiperiod securities markets, J. Econ. Theory 20, 381-408.

M. J. Harrison, S. R. Pliska (1981), Martingales and stochastic integrals in the theory of continuous trading, Stoch. Proc. Appl. 11, 215-260.

D. M. Kreps (1981), Arbitrage and Equilibrium in Economics with infinitely many Commodities, J. of Math. Econ. 8, 15-35.

S. Kusuoka (1992), A Remark on Arbitrage and Martingale Measure, to appear in Publ. RIMS Kyoto University.

P. Lakner (1992), Martingale Measures for Right-Continuous Processes which are bounded below, preprint, 9 p.

R. Merton (1973), The Theory of Rational Option Pricing, Bell Journal of Economics and Management Science 4, 141-183.

W. Schachermayer (1992), Martingale Measures for discrete time processes with infinite horizon, preprint, $30 \mathrm{p}$.

H. H. Schaefer (1971), Topological Vector Spaces, third printing, Springer Graduate Texts in Math.

C. Stricker (1990), Arbitrage et lois de martingale, Ann. Inst. H. Poincaré 26, 451-460.

M. S. Taqqu, W. Willinger (1987), The analysis of finite security markets using martingales, Adv. Appl. Prob. 19, 1-25.

W. Willinger, M. S. Taqqu (1988), Pathwise approximations of processes based on the fine structure of their filtrations, Sém. de Probabilités XXII, Lecture Notes in Mathematics 1321, 542-599. J.A.Yan (1980), Caracterisation d' une classe d'ensembles convexes de $L^{1}$ ou $H^{1}$, Seminaire de Probabilites XIV, Lect. Notes Mathematics 784, 220 - 222.

Institut für Mathematik der Universität Wien, Strudlhofgasse 4, A-1090 Wien, AusTRIA.

E-mail: wschach@stat1.bwl.univie.ac.at 